\title{
INTRODUCING CLEVIDIPINE IN PHAEOCHROMOCYTOMA SURGERY PROTOCOLS
}

\author{
J. Mendoza, A. López, J.A. Martínez-Molina, A. Figuerola, M. Sagardoy, A.D. González \\ Clínica Universidad de Navarra - Department of Anesthesia \& Intensive Care - Pamplona (Spain)
}

64 year old woman. Hypertension with hypertensive cardiopathy, left ventricle hypertrophy. Multinodular goitre, anxiety disorder. Chronic medication: Nifedipine 30mg (1-0-0); Venlafaxine 75mg (1-0-0); Alprazolam 0,25mg (0,5-0-0,5)

Addmission for laparoscopic adrenalectomy in the context of phaeochromocytoma.

\section{PREOPERATIVE MANAGEMENT:}

Because of blood pressure values over target $(120 / 60 \mathrm{mmHg})$, adrenergic bolckade with Doxazosine $30 \mathrm{mg}$ in the morning and volumen optimization with crystalloids because of orthostatic hypotension were required.

Surgery was delayed until blood pressure was controlled below $120 / 60 \mathrm{mmHg}$ values.

\section{INTRAOPERATIVE MANAGEMENT:}

Anesthetic induction with close blood pressure monitoring with an arterial cannula.

As the surgeon manipulates the tumor, a hypertensive peak (SBP $>180 \mathrm{mmHg}$ ) occurs.

We administered a continous infusion of Clevidipine with a máximum speed of $16 \mathrm{mg} / \mathrm{h}$. Once the tumor was removed, requirement of clevidipine drops to $4 \mathrm{mg} / \mathrm{h}$.

Hemodynamic stability was assured during the whole procedure.

\section{POSTOPERATIVE MANAGEMENT:}

Admission in the Intensive Care Unit.

Clevidipine infusión continues to be necessary for some hours (4mg/h).

The day after surgery, blood pressure values could be controlled with oral Nifedipine.

Discharge to regular hospitalization area with optimal blood pressure control. Habitual medication was reintroduced and patient was discharged home.

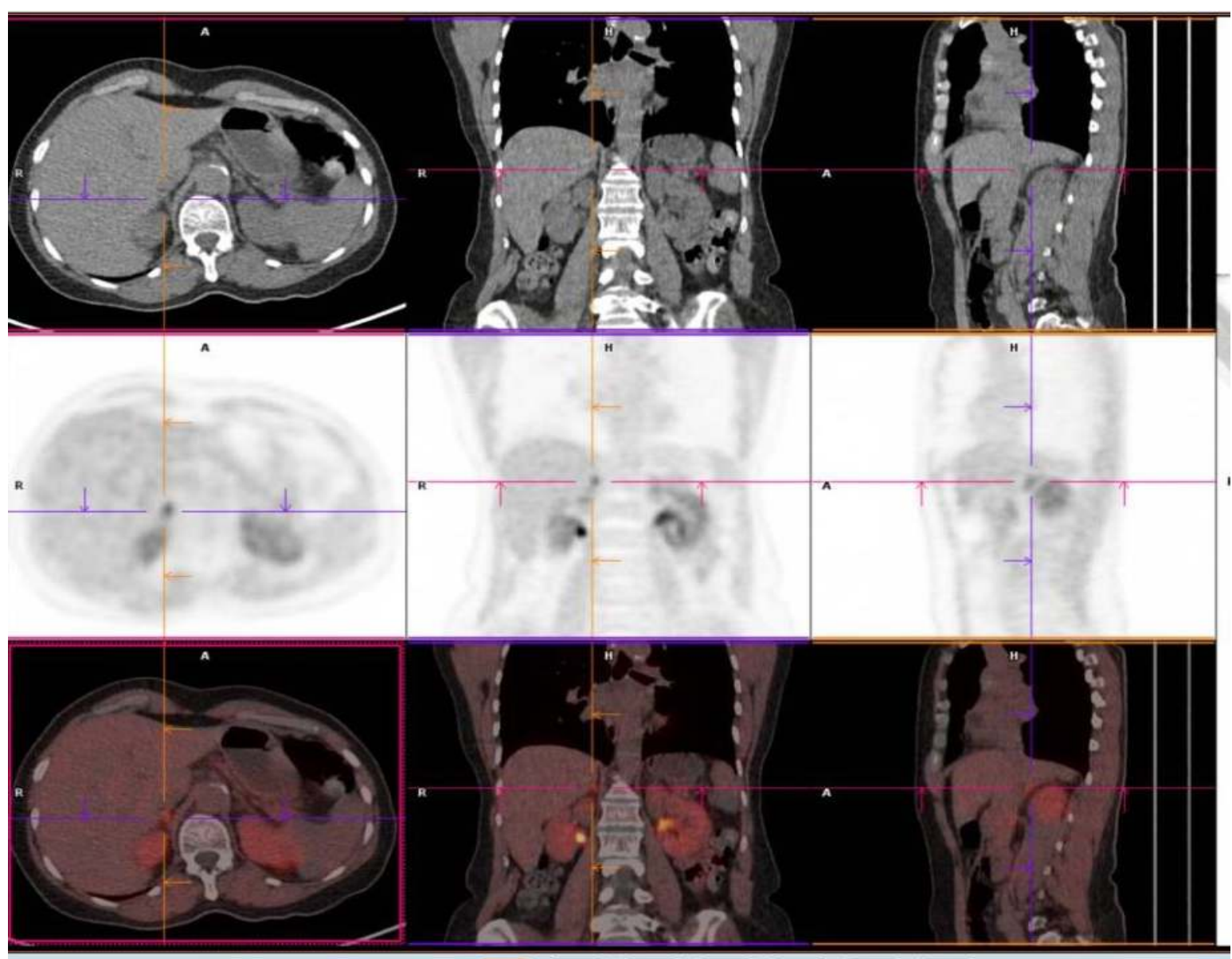

CLEVIDIPINE

MECHANISM OF ACTION:

4th generation dihydropyridine L-type calcium channel blocker.

Direct arterial vasodilatation with no effect on venous capacitance.

DRUG PROPERTIES:

Ultra-short half-life.

Rapidly metabolized by plasmatic esterases.

DOSING:

Starting dose $4 \mathrm{ml} / \mathrm{h}(2 \mathrm{mg} / \mathrm{h})$ titrated every $60-90$ seconds Dose Maximum $8 \mathrm{mcg} / \mathrm{kg} / \mathrm{min}$

Maximum $500 \mathrm{ml}$ in 24 hours

\section{DISCUSSION:}

Clevidipine continous infusion use in phaeochromocytoma surgery is not common. Clevidipine can be a new and very useful resurce to be introduced in this kind of surgery. Its rapid onset of effect and ultra-short half-life, make it a really interesting drug. On top of that, it has a very low incidence of adeverse effects and it can be easily titrated to get to our targeted blood pressure.

\section{REFERENCES:}

1. Naranjo J, et al. (2017), Perioperative Management of Pheochromocytoma. Journal of Cardiothoracic and Vascular Anesthesia, Volume 31, Issue 4, 1427-1439.

2. Augustides et al. Perioperative Managemet of Pheochromocytoma: Focus on Magnesium, Clevidipine, and Vasopressin. Journal of Cardiothoracic and Vascular Anesthesia, Vol 26, No 3 (June), 2012: pp 526-531

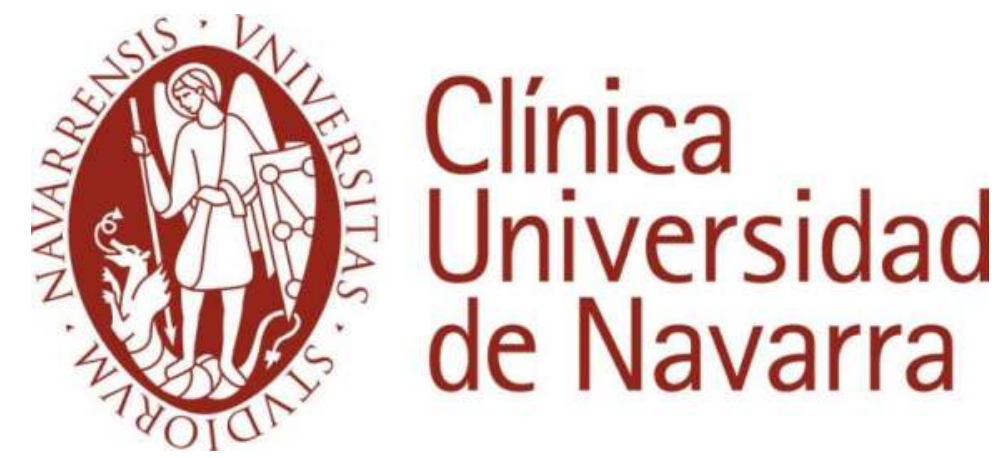

